\title{
Astrophysical roles for giant resonances in exotic nuclei
}

\author{
E. Khan ${ }^{\mathrm{a}}$ \\ anstitut de Physique Nucléaire, Université Paris-Sud, $\mathrm{IN}_{2} \mathrm{P}_{3}$-CNRS, \\ 91406 Orsay Cedex, France
}

An overview of the role of giant resonances in three different astrophysical schemes is given. These studies are possible thanks to the recent advent of microscopic QRPA models based on a single energy functional including pairing effects. First the existence of collective modes in the inner crust of neutron stars is discussed. Second, the dipole strength being a major component of the nuclear statistical (de-)excitation mechanism, it is crucial to predict the E1 strength on several hundreds of neutron-rich nuclei involved in the r-process, which can occur from a decompression of the neutron star crust. Finally a description for the propagation of the Ultra High Energy Cosmic Rays (UHECR), from an extra-Galactic source towards the Earth, is necessary in order to solve the mystery of their composition. UHECR may be composed of protons and nuclei lighter than Iron.

\section{Introduction}

It is likely to calculate the collective response with the Hartree-Fock-Bogoliubov + Quasiparticle Random Phase Approximation (HFB+QRPA) approach formulated in the coordinate representation [1] : the coordinate representation is suited to treat systems with a large number of quasiparticle states such as Wigner-Seitz cells in the crust of neutron stars. Moreover, predicting r-process abundances requires nuclear inputs from several thousands of nuclei [2]. Theoretical results play therefore a crucial role, and an universal description of this large variety of nuclei is necessary. The Skyrme HFB+QRPA approach allows to provide the dipole strength on the whole nuclear chart. Finally we will show that the dipole strength plays also a key role in the propagation of Ultra-High Energy Cosmic Rays.

\section{Supergiant resonances in neutron star crust}

The crust of neutron stars may collect one of the most exotic nuclear excitations. In microscopic calculations the inner crust matter is usually treated in the Wigner-Seitz (WS) approximation. Accordingly the inner crust is modelised by non-interacting cells containing a neutron-rich nucleus immersed in a dilute gas of neutrons and relativistic electrons. For baryonic densities ranging from $1.410^{-3} \rho_{0}$ to about $0.5 \rho_{0}$, where $\rho_{0}=0.16$ $\mathrm{fm}^{-3}$ is the nuclear matter saturation density, the nuclear clusters are considered spherical. A WS cells typically contains few tens of protons and few hundreds of neutron. For instance the cell with $\mathrm{Z}=50$ and $\mathrm{N}=1750$ is quoted as ${ }^{1800} \mathrm{Sn}$. In this cell the density of 
the neutron gas far from the nuclear cluster is of about $0.018 \mathrm{fm}^{-3}$ and the radius of the cell is $27.6 \mathrm{fm}$. As an illustrative picture, it can be considered as a system between a finite nucleus, and the uniform neutron matter. Nuclear collective modes are expected to develop in such WS cells containing spherical nuclear clusters.

The cooling of low-mass neutron stars is strongly influenced by the superfluid properties of inner crust matter [3]. The specific heat can also be strongly affected by the collective modes created by the residual interaction between the quasiparticles, especially if these modes appear at low-excitation energies. This effect has been studied in [4]. It should be noted that the specific heat of the inner crust is also largely determined by the electrons motion.

Within the HFB+QRPA formalism presented above [1], the responses of the ${ }^{1800} \mathrm{Sn}$ cell have been calculated for several multipolarities (Fig. 1). A very collective low-lying state is predicted, located in the $2-4 \mathrm{MeV}$ energy region. This mode typically takes $70 \%$ of the EWSR, which remains very high for a such collective state, so-called a SuperGiant Resonance (SGR) [4]. It is found that both contributions of the surface of the nuclear cluster and the external neutron gas are substantial. The unperturbed HFB response, built by non-interacting quasiparticle states, and the QRPA response are both shown in figure 1. When the residual interaction is introduced among the quasiparticles the unperturbed spectrum, distributed over a large energy region, is gathered in a peak located at about $3 \mathrm{MeV}$. All multipolarities exhibit a SGR, which can be more or less damped. This mode is extremely collective since there are more than one hundred twoquasiparticle configurations contributing. Another indication of the extreme collectivity of this low-energy mode can be seen from its reduced transition probability, B(E2), which is equal to $25.10^{3}$ Weisskopf units.
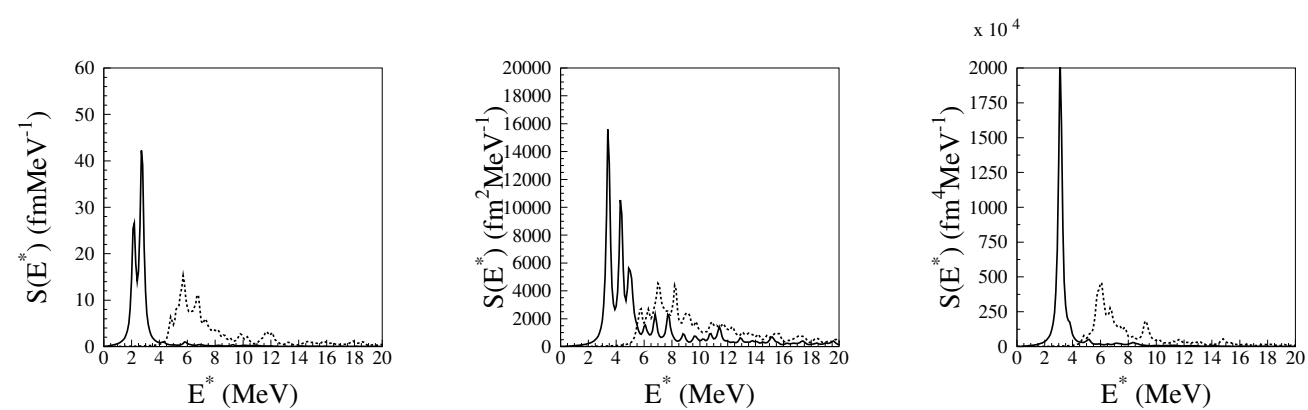

Figure 1. Monopole (left), dipole (center) and quadrupole (right) strengths of neutrons for the cell ${ }^{1800} \mathrm{Sn}$. The full curve represent the QRPA strength and the dashed line is the HFB unperturbed strength

\section{The r-process and the low-lying dipole strength}

About half of the nuclei with $A>60$ observed in nature are formed by the so-called rapid neutron-capture process (or r-process) of nucleosynthesis. Two main astrophysical 
sites may be candidates for hosting the r-process. The first one, which is commonly known, is occurring in explosive stellar events, such as core collapse supernova [2]. In this case, the r-process is believed to take place in environments characterized by high neutron densities $\left(N_{n} \simeq 10^{20} \mathrm{~cm}^{-3}\right)$, so that successive neutron captures proceed into neutron-rich regions well off the $\beta$-stability valley forming exotic nuclei that cannot be produced and therefore studied in the laboratory. If the temperatures or the neutron densities characterizing the r-process are low enough to break the $(n, \gamma)-(\gamma, n)$ equilibrium, the so-called waiting point approximation is not valid anymore, and the r-abundance distribution directly depends on the neutron capture rates by exotic neutron-rich nuclei [5].

An alternative r-process scenario is generating a renewed interest these last years. It is related to the decompression of cold neutron star matter, in particular its crust [2]. In this case the production of heavy nuclei follow a totally different path from the corecollapse supernovae scenario. The nuclear clusters immersed in the neutron gas are both ejected from the neutron star crust, inducing a decrease of the matter density. The $\beta$ equilibrium is broken, leading to the production of a variety of $\mathrm{Z}$, typically ranging from 40 to 70 . When the density reaches the drip density, drip-line nuclei are formed, which are immersed in a typical free neutron flux of $N_{n} \simeq 10^{35} \mathrm{~cm}^{-3}$. A competition between the neutron capture and the $\beta$ decay is then ignited from these drip-line nuclei, leading to the production of heavy nuclei. This alternative scenario shows also that nuclear inputs are necessary to modelise the r-process, since this nucleosynthesis path is initialized from the drip-line, where not waiting point approximation is possible.

Nuclear observables are therefore needed to predict r-process abundances. In addition to the $\beta$ decay, it is necessary to describe the $(\mathrm{n}, \gamma)$ rates, which can be divided in 2 steps : the neutron capture and the photodeexcitation of the nuclei [6]. The neutron capture rates are commonly evaluated within the framework of the statistical model of Hauser-Feshbach. This model makes the fundamental assumption that the capture process takes place with the intermediary formation of a compound nucleus in thermodynamic equilibrium. In this approach, the Maxwellian-averaged $(n, \gamma)$ rate at temperatures of relevance in r-process environments strongly depends on the electromagnetic interaction, i.e the photon de-excitation probability. The well known challenge of understanding the r-process abundances requires reliable extrapolations of the E1-strength function towards the neutron-drip line. It should be noted that soft dipole modes are expected to play an even more pivotal role for the r-process than the higher energy dipole strength. Lowenergy dipole modes located close to $S_{n}$ are directly fed by the one neutron capture reaction. The relevance of the soft dipole modes has been illustrated in ref. [5] where the presence of such states has a strong impact on the r-process.

Recently attempts were made to derive microscopically the E1-strength for the whole nuclear chart $[7,8]$. In a first step, the dipole response was calculated with the Quasiparticle Random Phase Approximation (QRPA) on top of Hartree-Fock+BCS (HFBCS) description [7]. In a second step, the HFB+QRPA model was used [8]. The only input of this approach was the Skyrme effective interaction injected in the HFBCS or HFB models. The comparison with the data shows that they not only reproduce extremely well the experimental masses (with a rms deviation as low as $0.676 \mathrm{MeV}$ on the 2135 known masses), but also are well adapted to describe the E1 collective excitations in the present approach. More precisely the agreement with the data is of the same accuracy than with 
phenomenological models, with a rms deviation around $500 \mathrm{keV}$ on the GDR position.

For astrophysical purposes, microscopic predictions need to be generalized on the whole nuclear chart, that is for odd and deformed nuclei. To achieve this goal, approximations are performed, keeping in mind that an order of magnitude accuracy is generally sufficient for astrophysical applications. The current approximation are : approximate treatment for odd nuclei, phenomenological damping of the E1 strength, and phenomenological treatment of the deformation. When dealing with neutron-rich nuclei, the QRPA predictions start deviating from a simple Lorentzian shape. In particular, some extra strength is found to be located at an energy lower than the GDR energy. The more exotic the nucleus, the stronger this soft dipole component. More generally, the HFB+QRPA calculation confirms that the neutron excess affects the spreading of the isovector dipole strength, as well as the centroid of the strength function.

The results of the Hauser-Feshbach calculations should be compared to experimental $(\mathrm{n}, \gamma)$ cross-sections. However it is much more convenient to measure the reverse $(\gamma, \mathrm{n})$ reaction. Such an experiment has recently been performed on ${ }^{181} \mathrm{Ta}$, allowing to complete the low-energy data of the ${ }^{181} \mathrm{Ta}(\gamma, \mathrm{n}){ }^{180} \mathrm{Ta}$ reaction [9]. Figure 2 shows the comparison between the data and the Hauser-Feshbach calculations, using either the QRPA E1 strength, or the phenomenological ones. At low energy, the microscopic treatment of the dipole strength gives significantly different results than the phenomenological approach. The tail of the cross section between 7.5 and $10 \mathrm{MeV}$ is due to the prediction of soft dipole modes in the QRPA calculations. It allows to describe very accurately the data, at variance with the phenomenological based calculations.

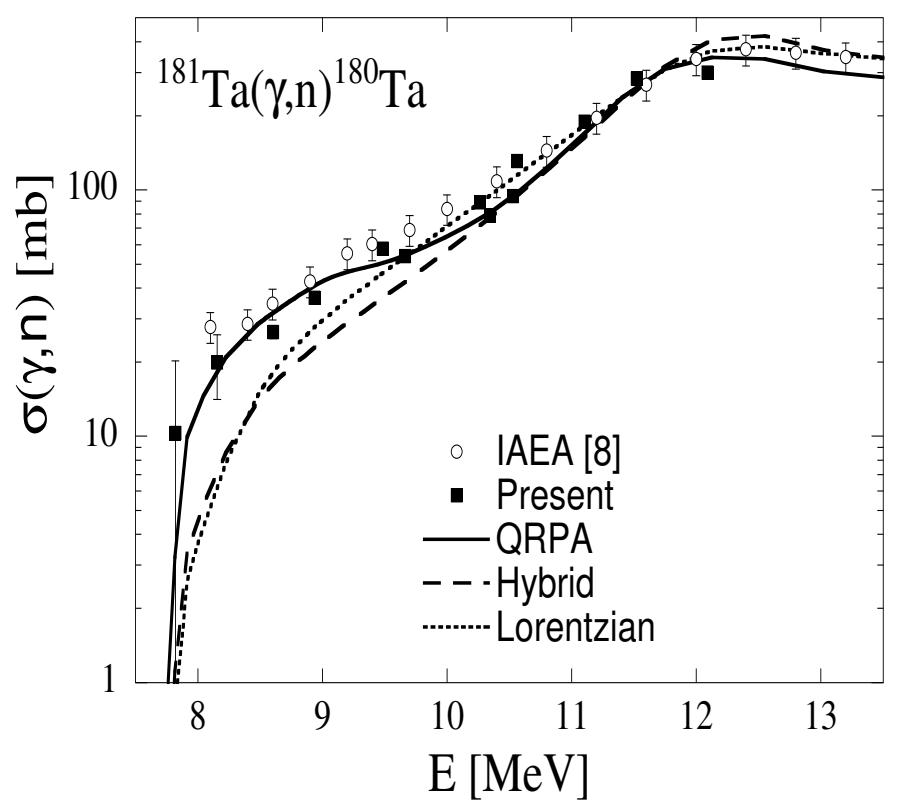

Figure 2. Comparison of the experimental photoneutron cross section for ${ }^{181} \mathrm{Ta}$ with the Hauser-Feshbach predictions obtained with the QRPA, Hybrid and Lorentzian models [9] 
The Maxwellian-average capture rates obtained with the QRPA E1-strength are compared in Fig. 3 with those based on the Hybrid phenomenological formula for all exotic neutron-rich nuclei with $8 \leq Z \leq 110$. The QRPA-derived E1 strength gives an increase of the rate by a factor up to 10 close to the neutron drip line. R-process nuclei characterized by $S_{n} \simeq 3 \mathrm{MeV}$ are seen to have a neutron capture rate about at least twice faster than the one predicted with the phenomenological Hybrid formula. This is partly due to the appearance of dipole modes at low energies as explained above. This effect tend to increase the E1 strength at energies below the GDR, i.e in the energy window of relevance in the neutron capture process. For less exotic nuclei, the QRPA impact is relatively small, differences being mainly due to the exact position of the GDR energy and the resulting low-energy tail. When compared to previous HFBCS+QRPA predictions [7], the HFB+QRPA model [8] gives very similar neutron capture rates even close to the neutron drip line, as seen in Fig. 3 (lower panel). This shows the consistency of the results obtained from microscopic approaches of the dipole strength.

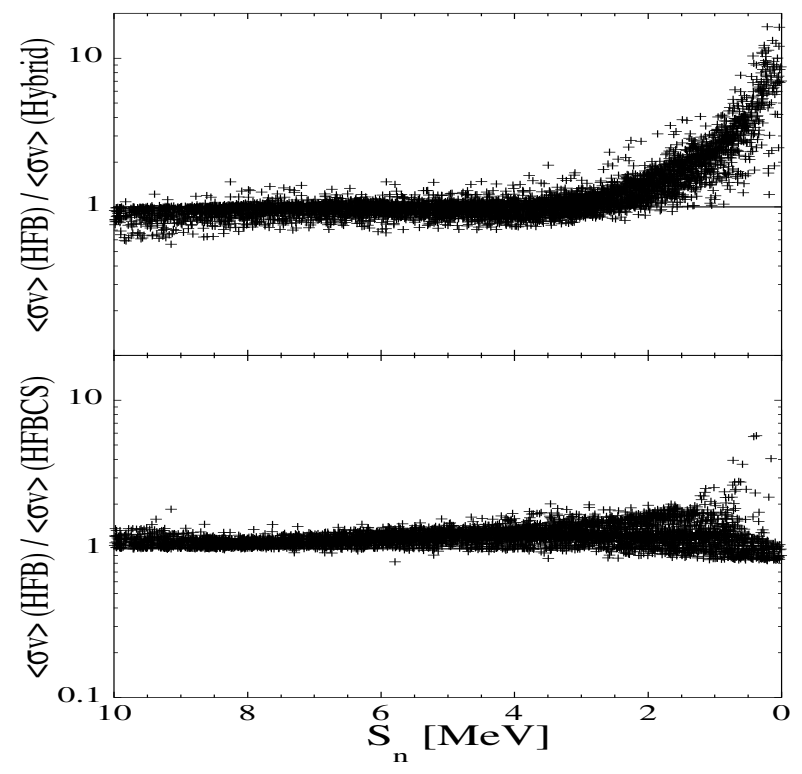

Figure 3. Upper panel: Ratio of the Maxwellian-averaged $(n, \gamma)$ rate (at a temperature of $1.510^{9} \mathrm{~K}$ ) obtained with the HFB+QRPA E1 strength to the one using the Hybrid formula [5] as a function of the neutron separation energy $S_{n}$ for all nuclei with $8 \leq Z \leq 110$. Lower panel: Same as upper panel where the HFB+QRPA neutron capture rates are compared with the HFBCS+QRPA rates of [7]. Taken from [8]

The deduced r-abundances have been calculated using the neutron star matter decompression scenario [2]. It shows a good agreement with the measured solar abundances for heavy nuclei $(A>120)$. Especially, the peak around $A=195$ is reproduced at the right position and with the right width. 


\section{Nuclei as Ultra-High-Energy Cosmic Rays}

Cosmic-rays have been observed up to energies of $\sim 310^{20} \mathrm{eV}$. Astrophysical sites able to accelerate particles to such ultra high energies are currently under discussion. Violent processes related to neutron stars are possible candidates since they generate sufficient magnetic fields to confine protons of $10^{20} \mathrm{eV}$. The composition of Ultra High Energy Cosmic Rays (UHECR) is also a puzzling mystery. Two main hypothesis have been advanced : protons, or a mixed composition of protons and nuclei from the Universe abundance. In this last case, nuclei ranging from proton to iron are mainly implied. It has been known for almost four decades that UHECRs interact with the $2.7 \mathrm{~K}$ cosmic microwave background radiation (CMB). Hence, UHECR issues can be decomposed in three steps : the initial composition, the acceleration mechanism, and the intergalactic propagation. Going through these steps, the UHECR may reach an Earth based detection setup such as the Auger detector [10]. The first two steps cover many unsolved questions about the composition and the acceleration processes of UHECR. It is therefore necessary to describe very accurately their propagation in order to provide a strong test on the composition and the acceleration scenarios by comparing the predictions with the data measured on Earth.

In the nucleus rest frame, at typical UHECR energies of $10^{19}-10^{21} \mathrm{eV}$, the CMB photons are boosted to energies in the range between a few hundreds of $\mathrm{keV}$ up to a few hundreds of $\mathrm{MeV}$. The interaction process between the UHECRs and the CMB is dominated by the giant dipole resonance (GDR) at photon energies below 30-50 MeV, and to a lesser extent by the quasideuteron emission for intermediate energies (between $50 \mathrm{MeV}$ and $150 \mathrm{MeV}$ ) and the pion photoproductions at energies above $150 \mathrm{MeV}$. Nuclei photodesintegrate by emitting nucleons through $(\gamma, \mathrm{n}),(\gamma, \mathrm{p}),(\gamma, 2 \mathrm{n}), \ldots$ reactions. It is therefore necessary to accurately describe the dipole strength for the involved nuclei during the photodisintegration path from Iron to protons. It should be noted that a large proportion of nuclei along this path are unstable, such as ${ }^{44} \mathrm{Ti}$.

To describe the changes in abundance of the heavy nuclei as a result of the interaction of the UHECR with the CMB, a nuclear reaction network including all interactions of interest must be used. The chosen set of nuclear species are coupled by a system of differential equations corresponding to all the reactions affecting each nucleus, i.e. mainly photodisintegrations and $\beta$-decays [11]. All nuclei lighter than the seed nuclei and located between the valley of stability and the proton drip line must be included in the network. Under the most natural astrophysical assumptions, UHECRs are accelerated out of the ambient gas, possibly enriched in Fe close to neutron stars or depleted in metals (i.e. nuclei heavier than $\mathrm{H}$ ) if significant photodisintegration occurs during the acceleration stage itself. Therefore, if nuclei are indeed present among the UHECRs, it is expected that they typically include the most abundant elements found in the interstellar medium, i.e. essentially lighter than Fe. The interaction of UHECRs with the CMB is thus expected to include all possible nuclei resulting from the photodisintegration of the heaviest species and therefore involve all stable and neutron-deficient unstable isotopes with $\mathrm{A} \leq 56$.

The photoreaction cross sections are estimated with the Talys nuclear reaction code [12] which takes into account all types of direct, pre-equilibrium and compound mechanisms to estimate the total reaction probability as well as the competition between the various open 
channels. It should be recalled that, even for stable nuclei, the data on such nuclei are scarce. For instance, total photoabsorption cross sections around the GDR peak energy are available for only 10 nuclei [13]. An exhaustive comparison has been performed to compare the predictions with the data [11].

To illustrate the role of the soft dipole mode, let us consider a ${ }^{56} \mathrm{Fe}$ nucleus with an energy of $E=3.6 \times 10^{20} \mathrm{eV}$. In this regime, only the lowest energy part of the $E 1$ strength overlaps with the photon density $\mathrm{n}(\epsilon)$. The distance of propagation is mainly sensitive to the low-energy component of the E1-strength function and the use of different prescriptions leads to significant differences in the propagation distance. This stresses the necessity to provide an accurate description of the low energy tail of the E1 strength, even for nuclei close to the stability. In contrast, results on ${ }^{56} \mathrm{Fe}$ at higher energy mainly depend on the GDR peak location or integrated photoabsorption, and for this reason the propagation distance is less sensitive to the photoreaction details.

The UHECR propagation distance has been estimated making use of the full reaction network. The initial calculations were based on a reduced path illustrated in Fig. 4. In this approximation, only one stable isotope is considered per isobaric chain and the corresponding isobars (i.e. nuclei with the same A) are not affected by competitive channels. However, as shown in Fig. 4, about 85 nuclei are involved in the ${ }^{56} \mathrm{Fe}$ photodisintegration at $\mathrm{E}=10^{21} \mathrm{eV}$ and numerous open channels including $\beta$-decay can compete (the Lorentz dilation of time allows $\beta$-unstable nuclei with half-lives of the order of the hour to survive over a Mpc scale, and thus have a chance to interact with a CMB photon). Most of the stable nuclei involved in the photodisintegration process have more neutrons than protons. Neutron emissions are therefore favored and the corresponding unstable nuclei will $\beta^{+}$-decay towards the valley of stability.

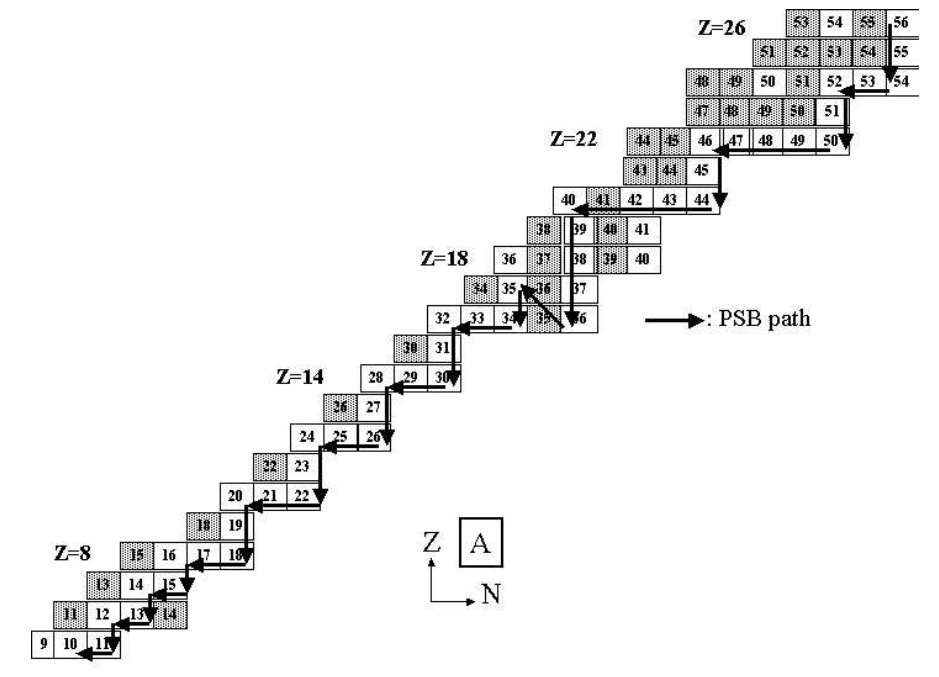

Figure 4. Nuclei involved in the photodisintegration process of ${ }^{56} \mathrm{Fe}$ nuclei for $E=10^{21} \mathrm{eV}$ Unstable nuclei are in shaded squares, and the former considered path is indicated by the arrows. The mass number of each nucleus is written in the corresponding square. [11] 
A full propagation calculation have been presented in Ref. [14]. A mixed source of protons and nuclei with $\mathrm{A}<56$ is considered, as well as quasideuteron, pion photoproductions and pair productions processes. The results of this Monte-Carlo simulation can compared with the cosmic ray data, and shows a good reproduction.

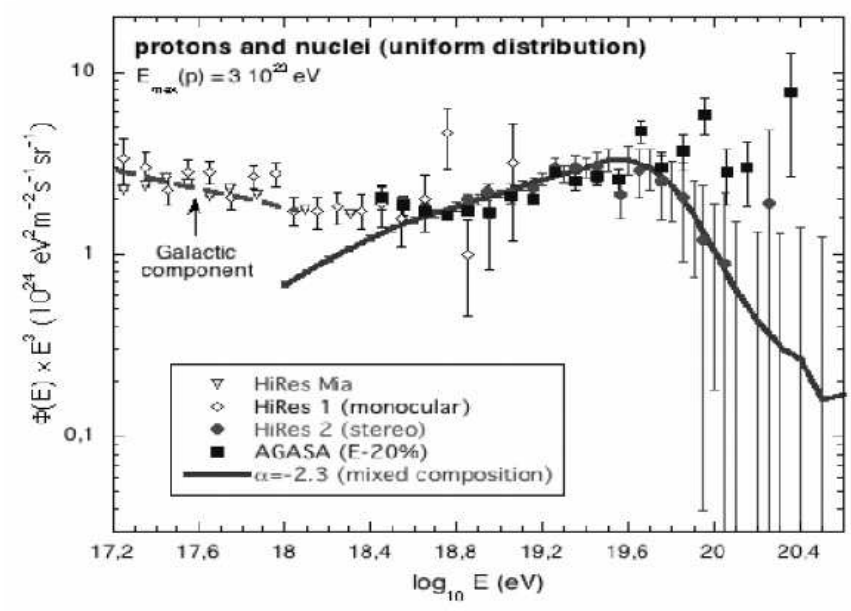

Figure 5. Propagated spectra from a source composed of protons and nuclei (solid line), compared with the available data [14]

It has been also shown in ref [14] that a mixed composition of protons and nuclei provide a solid interpretation of the high energy part of the cosmic ray spectrum around the socalled ankle area, and is also more in agreement with source models than with a uniform proton source. Moreover, ref. [15] have showed that considering nuclei in UHECR allows to reproduce complementary experimental observables in much better agreement than with protons only. This set of recent studies make a strong point to consider UHECR as composed of nuclei.

\section{Conclusions}

Collective modes are microscopically investigated in several astrophysical scenario, using Skyrme HFB+QRPA model. In the neutron star crust, the predicted energies of the SGR have the same order of magnitude as the average pairing gap of neutron superfluid. Consequently, these collective modes can affect significantly the entropy and the specific heat of baryonic inner crust matter. A quantitative estimation of these effects would require finite-temperature HFB+QRPA calculations, and will certainly be undertaken in the near future. Questions remains about the validity of the non-interacting WS approximation to describe collective excited modes. The relevant cells which have an impact on the specific heat remain also to be determined. Finally, neutron-rich nuclei produced by the next decade facilities may provide data in order to constrain models and functionnals 
describing WS cells. An identification of these key nuclei should be performed.

In the case of the r-process and UHECR propagation, many further improvements may be useful, which call for intensive theoretical developments, such as large scale microscopic deformed QRPA predictions of the E1 strength. The particle-vibration coupling affects the low-energy strength and could also contribute to an extra increase of the radiative neutron capture rate by exotic nuclei. Finally, a more suited treatment for odd nuclei should also be undertaken. The long range goal of such calculations is to fully microscopically describe the r-process or the photodisintegration path of UHECR with a single nuclear functionnal as input.

\section{REFERENCES}

1. E. Khan, N. Sandulescu, M. Grasso, Nguyen Van Giai, Phys. Rev C66 (2002) 024309.

2. S.Goriely, P. Demetriou, H.-Th. Janka, J.M. Pearson and M. Samyn, Nucl. Phys. A758 (2004) 587.

3. D. G. Yakovlev and C. J. Pethick, Annu. Rev. Astron. Astrophys 42 (2004)169

4. E. Khan, N. Sandulescu, Nguyen Van Giai, Phys. Rev. C71 (2005) 042801

5. S.Goriely, Phys. Lett. B436 (1998) 10.

6. S.Goriely and P. Descouvement, Ecole Int. Joliot-Curie (2000)

7. S.Goriely, E. Khan, Nucl. Phys. A706 (2002) 217.

8. S.Goriely, E. Khan and M. Samyn, Nucl. Phys. A739 (2004) 331 .

9. H. Utsunomiya, H. Akimune, S. Goko, M. Ohta, H. Ueda, T. Yamagata, and K. Yamasaki,H. Ohgaki,H. Toyokawa,Y.-W. Lui,T. Hayakawa, T. Shizuma, E. Khan, S. Goriely, Phys. Rev. C67 (2003) 015807.

10. P. Mantsch, 29th Int. Cosmic Ray Conf. (2005), Pune, India, astro-ph/0604114.

11. E. Khan, S. Goriely, D. Allard, E. Parizot, T. Suomijarvi, A.J. Koning, S. Hilaire, M.C. Duijvestijn, Astroparticle Phys. 23 (2005) 191.

12. A.J. Koning, S. Hilaire and M.C. Duijvestijn, Proceeding of the International Conference on Nuclear Data for Science and Technology (Santa Fe, USA), 2004.

13. Photonuclear data for applications; cross sections and spectra, IAEA-Tecdoc-1178 (2000).

14. D. Allard, E. Parizot, A.V. Olinto, E. Khan, S.Goriely, Astr. and Astr. 443 (2005) L29.

15. D. Allard, E. Parizot, A.V. Olinto, in preparation, astro-ph/0512345 (2006). 